\title{
PAGO POR SERVICIOS AMBIENTALES \\ EL CASO DE LA BELLEZA ESCÉNICA DE Araucaria Araucana Mol. Koch
}

Jorge Cabrera' y Marco Rubilar ${ }^{2}$

\section{RESUMEN}

Los bosques de Araucaria araucana se encuentran declarados Monumento Natural que prohíbe su corta y comercialización, razón por la cual muchos de estos bosques, principalmente privados y en manos de pequeños propietarios, se encuentran abandonados y en proceso de degradación.

Como una forma de buscar nuevos sistemas de protección de la especie, el presente estudio diseña un sistema de pago por servicios ambientales, basado en el servicio de la belleza escénica de la especie que en pequeños parques puede brindar.

Metodológicamente este trabajo se concentra fundamentalmente en determinar la disposición a pagar por el servicio, utilizando para ello el método de valorización contingente. Basado en un muestreo prospectivo, un instrumento de toma de datos, proceso de consulta directa y procesamiento estadistico económico, se obtuvieron cifras de disposición de pago positivas. Por parte de los estudios de la oferta, éstos se redujeron a una ronda de visitas más que a un muestreo estadistico debido a la claridad de la situación actual de los propietarios de araucaria.

En base a estos antecedentes la factibilidad técnico económica de implementar un Pago por Servicios Ambientales se califica como alta.

Palabras claves: Araucaria araucana, pago por servicios ambientales.

\section{SUMMARY}

Araucaria forests (Araucaria araucana) are under protection as National Monument in Chile, so harvesting and trade are not allowed. Conservation is ensured, but many Araucaria forests, mainly belonging to private and small owners, are currently neglected and exposed to degradation processes.

As a way to find alternatives to protect the species, an environmental services payment system design, based on the scenic value of the species in small parks, is the main objective of the work described on this paper.

\footnotetext{
'Ingeniero Forestal M Sc. Sede Valdivia. Instituto Forestal jcabrera@infor.cl

2 Ingeniero Forestal. Universidad de Concepción.
} 
Regarding to the methodology, the paper focused on the willingness to pay for the service determination, using for that the contingent valuation methodology. Based on a prospective sampling, a data collect instrument, a direct consultation process and a statistical method, positive willingness to pay figures were obtained. The current situation of the Araucaria forest owners is quite clear, so statistics sampling was not necessary, several visits to them were enough.

Based on the above background, the technical and economic feasibility to the Environmental Services Payment System setting up was considered as high.

Key words: Araucaria araucana, Environmental Services

\section{INTRODUCCIÓN}

Según la normativa chilena, no se puede usufructuar, a travès del uso comercial este árbol nativo de la zona sur del continente sudamericano. La gran mayoria de las zonas forestales con concentraciones, de cualquier nivel, de araucarias se encuentra bajo el firme alero de protección gubernamental, ya sea como Monumento Natural o como Reservas Forestales y Parques Nacionales (SNASPE).

En particular, la especie sólo podria ser explotada para uso cientifico, pero no comercial, bajo una estricta justificación dentro de un plan de manejo que debe aprobar la autoridad forestal.

La otra cara de esta situación la conforman los dueños privados de terrenos con este tipo de bosques. El poseer una araucaria dentro de los limites geográficos de un terreno privado significa que a este suelo no se le puede asignar ningún otro fin que mantener con vida a dicho árbol. Si algún agente privado talara una araucaria sufriria de los rigores de la ley con sus respectivas multas.

De esta forma el gobierno ha diseñado un mecanismo de protección coercitivo e intimidador para la protección de esta especie nativa de la tala para fines comerciales. Sin embargo, esto no garantiza su protección y mas bien genera incentivos perversos sobre la especie (Rubilar, 2008).

Sin embargo, este mecanismo no goza de perfección, por el contrario, se transforma en un incentivo para que privados no la protejan, pues ha perdido su valor económico para ellos, y para que otros con intenciones inescrupulosas construyan métodos perversamente elegantes. El más conocido de ellos, es el de quemar o incendiar árboles sanos dejándolos en un estado tal que se pueda terminar de eliminar del suelo y utilizarse éste para "mejores" fines.

Para el presente trabajo, se asume que el beneficio ambiental por parte de la araucaria seria su aporte en la belleza ambiental de su propio entorno. La literatura (Landell-Mills et al., 2002; Wunder, 2005; Wunder et al., 2008; Wünscher et al., 2007) reconoce varios servicios más, pero para este caso no serán de interés, sino solo la belleza escénica. Las razones que se 
esgrime se sustentan en dos aspectos, en primer término su distribución geográfica y altitudinal y, en segundo, su lento crecimiento.

En este trabajo se revisa un conocido método directo microeconómico-ambiental de preferencia extraida como es la valoración contingente, pero en su versión más ampliada y atrevida como es el Pago por Servicios Ambientales, aplicado a una especie arbórea nativa. Se examina un método que arroje un resultado formal para la estimación de los parámetros que defina necesarios, tanto para quien provea el servicio ambiental como para quienes lo utilicen.

La idea es generar un mercado ficticio para el servicio ambiental entregado por la araucaria, a saber, la belleza escénica, mediante una estimación de la disposición a pagar (Dp) y la disposición a aceptar (Da), calculando el equilibrio del mercado bajo algunos supuestos estilizados. Se caracterizará la demanda y la oferta para dar forma a este mercado ambiental, buscando que las mismas fuerzas del mercado lleven a la asignación de un precio eficiente para la belleza escénica de la araucaria. Antes de todo esto se realiza una revisión de literatura sobre el PSA, agrupando conceptos, ideas y resultados de investigaciones anteriores. Luego, se comenta sobre la metodología que se aplica en la caracterización de los lados del mercado, dado que describir la oferta es una tarea completamente diferente a la de describir la demanda en este contexto, por su tamaño y perfil. Finalmente, los resultados son presentados en conjunto para observar su relación. Desde el inicio del trabajo se percibe la necesidad de profundizar en PSA en el tema de la araucaria, por ser una especie tan sensible en Chile, así como en otras áreas susceptibles o de riesgo bioeconómico.

\section{OBJETIVOS}

Diseñar un pago por servicios ambientales donde especificamente se pretende:

- Determinar si existe un trade-off entre los bosques protegidos de araucaria y la demanda por beneficios ambientales.

- Valorar económicamente el bosque de araucaria utilizando el método de valoración contingente.

- Caracterizar el nivel de aceptación de implementar un proyecto de PSA para belleza escénica en un parque de araucaria por parte de los propietarios.

\section{MATERIAL Y MÉTODOS}

\section{El Pago por Servicios Ambientales: Una Revisión}

EI Pago por Servicios Ambientales, PSA en adelante, es un innovador instrumento para financiar la conservación de la naturaleza y manejar los recursos naturales. Se conoce como el pago directo por el mantenimiento o provisión de los servicios ambientales por parte de los usuarios del servicio, el cual se destina a los proveedores. La idea del PSA es crear un mercado para un bien que habitualmente no tiene precio, persiguiendo la asignación eficiente de los recursos. 
Su utilización está ampliamente documentada en aplicaciones realizadas en pequeños paises en desarrollo, en especial latinos. Depende de la clara identificación de los agentes, proveedores y usuarios, y de la relación entre uso de tierra y provisión de los servicios.

Este método contingente es fundamentalmente diferente de otros enfoques de conservación. En lugar de que otros presuponen que todos ganan, este enfoque reconoce explicitamente un fuerte trade-off en el montaje de paisajes con el uso de la tierra y trata de conciliar el conflicto de intereses, a través de una indemnización. Si bien existen sistemas PSA en algunas economias desarrolladas, siguen siendo mal aplicados en los paises en desarrollo. Hay muchas iniciativas incipientes en esto (e.g. Landell-Mills et al., 2002; Pagiola et al., 2002).

Cuatro tipos de Servicios Ambientales (SA) actualmente son destacados:

- La captura y almacenamiento de carbono y provisión de aire.

- La protección de la biodiversidad.

- La protección de cuencas hidrográficas y provisión de agua.

- La belleza escénica.

A veces, varios servicios pueden ser proporcionados en forma sinérgica como un 'sistema' de régimen de pago, que puede permitir que varios usuarios de los servicios ambientales paguen por sus servicios a varios proveedores de servicios en forma simultánea. La solución de este problema no es obvia, dado que no todos los bienes ambientales calzan fácilmente en un programa de servicios ambientales y no todas las personas están dispuestas a pagar por ellos. También se puede detectar parciales trade-offs entre los servicios. Además, algunos servicios ambientales, diferentes de los mencionados anteriormente, podrian ser objeto de comercio (e.g, áreas silvestres que proporcionan servicios de polinización a la agricultura), pero esta situación es muy poco observada.

Las conclusiones sobre este método no son homogéneas (Romero y Andrade, 2004; Karsenty, 2004; Vogel, 2002). Por un lado el PSA puede provocar financiamiento para comunidades pobres, a través de la venta de estos servicios, pero por otro lado puede generar mayor desigualdad de la distribución económica si en este programa participan grandes holdings que pueden privar a las comunidades de sus derechos legitimos. Además, algunos opositores a los PSA tienen intereses creados. Para un donante, el dinero que pasa de la mano del demandante al oferente, obviamente, ofrece menos oportunidades de publicidad que un multifacético proyecto de desarrollo rural. En esta fase, la incorporación del PSA en los paises en desarrollo probablemente se enfrenta a dos obstáculos claves y una barrera de comunicación.

El primer obstáculo es la limitada demanda; muy pocos usuarios de los servicios están seguros sobre el mecanismo para el que "estarian" dispuestos a pagar, en algunos casos, debido a que el vinculo entre el uso de la tierra y la disposición del SA es insuficientemente comprendido o ambiguo. El segundo obstáculo es la escasez de conocimientos sobre la 
dinámica de la oferta de Servicios Ambientales (SA). Cuando hay demanda de SA y se conoce su disposición a pagar, la pregunta es: ¿Cuáles son las condiciones previas institucionales necesarias para que los proveedores de PSA negocien un acuerdo? Demasiado poco se sabe y son necesarias más manos de experimentados. Por último, comunicar el concepto del PSA es un problema. Los partidarios suelen utilizar una base económica, mientras que los escépticos se basan en otras ciencias sociales (antropologia, sociologia, psicologia, ciencias politicas). Obviamente, hay margen para mediar entre estas dos ciencias. Formalmente, la literatura no ha presentado una definición única sobre este tema, generando en ocasiones confusión. Sobre este mismo tema, Wunder (2005) presenta en cinco puntos lo que sería la conceptualización del pago por servicios ambientales, dada su propia experiencia.

PSA es una transacción voluntaria, donde:

- El servicio ambiental está bien definido.

- Es comprado por un consumidor de servicios ambientales.

- Desde un proveedor de servicios ambientales.

- Si y sólo si el servicio ambiental es provisto con seguridad.

- Es un pago condicionado a una actividad de conservación.

En su correcto diseño es necesario, identificar los agentes que son, por un lado, los responsables de la externalidad positiva, proveedores del recurso, y por otro lado los usuarios del recurso. EI PSA es un mecanismo que enmarca una "negociación voluntaria", que viene de las medidas de comando y control, donde se supone que los potenciales proveedores del servicio ambiental pueden darle realmente un uso alternativo al suelo que les pertenece. Lo que se compra tiene que estar bien definido. La literatura comenta (Wunter, 2005) que el servicio ambiental puede ser medible tanto en forma directa como indirectamente, como el uso de la tierra para proporcionar un servicio.

También es necesario considerar los factores exógenos a la implicancia del SA, ya que la misma naturaleza no tiene un comportamiento constantemente predecible. Por ejemplo, si se quiere otorgar el servicio de limpieza del agua, a través de la conservación de los bosques, este resultado se puede ver afectado por tormentas tropicales afectando dominantemente la calidad del agua. Se ha comprobado que algunos pagos por SA, aunque sean fundamentados en relaciones improbables o en mitos, pueden permanecer por mucho tiempo. A veces, incluso, no se tiene la certeza de que los SAs clasifican como reales o "imaginarios". Pese a esto, se sostiene que una mala base para el PSA radicará en una baja solidez y sostenibilidad, dada la incertidumbre que se podria generar en el demandante del servicio, implicando finalmente la eliminación de sus pagos.

En cualquier PSA, debe haber recursos que van desde al menos un comprador del SA a por lo menos un proveedor, aunque la transferencia se produce a menudo, a través de un intermediario. Por último, pero no por ello menos importante, el régimen de PSA depende 
de cuanto pague el usuario, de la cuantia de prestación del servicio ambiental y que ésta sea continuo. Por consiguiente, los compradores de SAs suelen vigilar el cumplimiento del programa y si éste se apega a lo que el contrato firmado ha estipulado. En los paises desarrollados, el apoyo legal puede crear las condiciones de confiabilidad para el flujo seguro de los pagos (e.g. Bayon, 2004; Sokolow y Zurbrugg, 2003). Como es de suponer, en los paises en desarrollo el marco legal no existe o es incipiente. Esta característica implica que el PSA normalmente debe ser controlado periódicamente, a menudo con un horizonte infinito, atado a un seguimiento del cumplimiento. Por lo tanto, los compradores de servicios deben tener la posibilidad de retirarse de un contrato PSA si no reciben lo que pagan. Por el contrario, los proveedores de servicios también puede tener un interés de flexibilizar los contratos, a fin de que puedan salir (o modificar los términos) de un régimen PSA si las condiciones del contexto cambian (Wunter, 2005).

El asunto de mantener el flujo seguro de los pagos es la condición más difícil de cumplir. Se dice que muchas iniciativas de PSA son poco controladas, los pagos son por adelantado en vez de posteriores o periódicos y sólo se realizan de buena fe, sin gozar de un verdadero seguimiento. Los Pagos por Servicios Ambientales podrian proporcionar una fuente alternativa de ingresos para los dueños de bosques, mientras que se mantengan los servicios forestales (belleza escénica). Para esto, se recuerda, requiere una buena comprensión de la interacción entre los agentes.

A continuación se trata sobre la eficacia y la eficiencia del diseño del PSA en un contexto de explotación inmoral de los derechos de propiedad y en presencia de intereses comerciales. Para la eficacia, se requiere que el PSA lleve un aumento real de los servicios ambientales en comparación con la situación que se produciria sin PSA. La eficiencia se refiere a aprovechar al máximo los servicios ambientales obtenidos a partir de un determinado presupuesto. El diseño eficaz y eficiente del PSA requiere, entre otras cosas, de la estimación del costo de oportunidad de los dueños de araucaria, para el presente caso de estudio.

¿Cuáles son las fuentes de esta variación y cómo se puede estimar los beneficios esperados para una comunidad que se está considerando para PSA? Entre los investigadores del PSA, la intuición común parece sugerir que, para un determinado servicio ambiental por hectárea, el PSA se deberia centrar en los costos de oportunidad más bajos de los dueños de bosques nativos (e.g. Siikamäki y Layton, 2006; Wúnscher et al., 2006).

Se puede esperar tres resultados. Por un lado que algunos dueños tengan costo de oportunidad tan bajos que la aplicación de un PSA por parte de los vecinos que pagan sería un gasto más pequeño que el mismo costo de implementación de la politica. Por otro lado, el costo podria ser tan alto que no sea politicamente implementable y, finalmente, que sólo por un asunto "moral" los dueños de araucarias jamás quemarian sus posesiones para asignarle al suelo un uso alternativo.

Yendo a otro tema, existen en forma general dos tipos de PSA:

- Utilización de los instrumentos de mercado para el pago de servicios cuyos usuarios no están restringidos a nivel local. 
- Compensación de proveedores, a través de un mercado laboral. Aquí los usuarios están mejor circunscritos al área de la actividad productiva.

De Hek (2004) destaca que su principal problema son los costos de transacción, como la elaboración de un estudio biofísico, la valoración económica y otros. Además ha detectado que cuando los recursos que se obtienen para la implementación de un PSA no pertenecen a la localidad, este programa genera dependencia de esos recursos y nunca se liberan para trabajar independientemente, por lo que se aconseja que se utilicen recursos locales para que sea sostenible en el largo plazo. El mismo autor comenta que los PSAs sirven para la sensibilización de los agentes y que un marco legal no es tan necesario para la implementación del PSA, sino la confianza entre el proveedor y usuario y un buen intermediario. Cuando el programa de PSA trabaja en una localidad pequeña y claramente enfocado a un sector, se ha detectado mayor impacto que cuando el programa es destinado a un sector amplio y generalizado (De Hek, 2004). Se recuerda que para evaluar la factibilidad del PSA es necesario realizar un estudio de demanda y oferta de los servicios ambientales.

Por la falta de conocimiento teórico explícito sólido y una buena base empírica se ha detectado algunos mitos, de los que se pueden mencionar algunos (Cuadro $\mathrm{N}^{\circ} 1$ ).

\section{Cuadro $N^{\circ} 1$ \\ MITOS Y REALIDADES SOBRE EL ROL DEL BOSQUE}

\begin{tabular}{|c|c|}
\hline Mitos & Posible Realidad \\
\hline $\begin{array}{c}\text { La reforestación aumenta la disponibilidad } \\
\text { de agua. }\end{array}$ & $\begin{array}{c}\text { La reforestación puede disminuir la } \\
\text { disponibilidad de agua. }\end{array}$ \\
\hline $\begin{array}{c}\text { La cobertura vegetal reduce la probabilidad } \\
\text { de grandes inundaciones. }\end{array}$ & $\begin{array}{c}\text { La cobertura vegetal puede tener poco } \\
\text { efecto sobre las grandes inundaciones. }\end{array}$ \\
\hline $\begin{array}{c}\text { La reforestación reduce la erosión. } \\
\text { causas más importantes del incremento de } \\
\text { la sedimentación. }\end{array}$ & $\begin{array}{c}\text { La reforestación puede aumentar la erosión } \\
\text { (dependiendo de la especie). }\end{array}$ \\
\hline Los bosques incrementan la precipitación. & $\begin{array}{c}\text { Las variaciones climáticas pueden ser la } \\
\text { causanportante del incremento de la } \\
\text { sedimentación. }\end{array}$ \\
\hline & $\begin{array}{c}\text { Los bosques pueden tener un efecto } \\
\text { ponsiderable sobre la precipitación, } \\
\text { particularmente a escala local. }\end{array}$ \\
\hline
\end{tabular}

(Fuente: De Hek, 2004).

Además de estos mitos, investigaciones a priori han presentado los siguientes errores:

- El uso de fuentes secundarias para otorgar valores de mercado (e.g. estudios de Valoración Contingente de otros lugares). 
- Valorar el monto total del recurso en una zona (e.g. valorar un bosque), cuando lo que interesa conocer es el efecto marginal sobre el servicio ambiental producto de un cambio en el uso del suelo.

- Tendencia a valorar los beneficios esperados del cambio en el uso del suelo, pero a ignorar los costos asociados a dichos cambios.

Los PSA no necesariamente involucran pagos de dinero en efectivo, sino pueden ser incentivos fiscales, pago en bienes, capacitación, crédito o algún otro tipo. También estos pagos por servicios ambientales pueden ser individuales o colectivos. Para poder motivar al proveedor de los servicios a conservar los recursos naturales, el PSA deberia pagarle de manera tal que pudiese percibir al mismo nivel que si produjera rentablemente en su finca.

El mecanismo para la gestión del PSA se recomienda sea una entidad multi-actor en que participen representantes de cada uno de los sectores involucrados, como una autoridad de protección de los bosques, que incluye representantes del gobierno, instituciones privados y ONG, con procedimientos que aseguren transparencia e imparcialidad.

El Gobierno puede ser el que facilite la interacción entre los actores privados, entregando un marco jurídico adecuado para que los PSA estén adecuadamente reglamentados y estableciendo los montos a pagar en base a estudios técnicos y acuerdos entre los actores relevantes. También puede jugar el rol regulador para no dejar al libre mercado el manejo de los recursos financieros para el medio ambiente.

\section{Estimación del Mercado}

Se aplican técnicas de valoración económica para bienes sin mercado para estimar el comportamiento de los dos lados del mercado. Primero se estimará la Disponibilidad a Aceptar (Da) un pago para proteger las araucarias por parte de los dueños, i.e. la oferta del mercado. Segundo, se estimará la Disponibilidad a Pagar (Dp) por mantener la belleza escénica otorgada por araucarias en manos de privados, es decir, la caracterización de la demanda del mercado. Tercero, utilizando el análisis de los elementos anteriores se determina la factibilidad de implementar un PSA considerando opciones y limitantes.

\section{-Valorización Contingente: Disponibilidad a Aceptar: Caracterización de la Oferta}

En la construcción del mercado hipotético, los agentes que definen el lado de la oferta están constituidos por los dueños de los suelos contenedores de las araucarias. Estas personas tienen la caracteristica de ser una población notablemente menor en relación a quienes son los que demandan los servicios ambientales. Como se dijo anteriormente, la oferta agregada de los servicios ambientales para preservar el suelo que contenga araucarias, estaria constituida por la suma horizontal de todas las ofertas individuales de servicios ambientales de cada uno de los oferentes, donde la cantidad ofrecida se encuentra en el eje de las ordenadas y el precio en el eje de las abscisas. La población alcanza un grupo total de 5 individuos los cuales serán caracterizados, a través de encuestas que determinen 
su Disponibilidad a Aceptar ${ }^{3}$ una compensación pecuniaria para disponer sus terrenos para la preservación de esta especie arbórea nativa.

Para comenzar a formalizar el método para clarificar el perfil del oferente, es necesario considerar que no es necesario desarrollar una estimación del comportamiento de la oferta, esto porque el tamaño de la población es tan reducido que estadísticamente, como se indicó en términos discretos, no existe un tamaño de muestra suficientemente grande como para que entregue resultados significativos y consistentes. Por ello, se recurrió a sondear toda la población en cuestión, obteniendo resultados absolutamente certeros, dado el instrumento de obtención de información, sin tener la necesidad de asignar una probabilidad de significancia a las conclusiones. Luego, los resultados obtenidos de un censo son ampliamente aceptados, tanto en términos estadísticos como económicos.

Se generó una encuesta construida consistente con las recomendaciones del panel NOAA (National Oceanic Atmospheric Administration) de tal manera que el instrumento sea creible, de acuerdo a como lo condujo el investigador, e.g. la encuesta fue en persona, el encuestado recibió toda la información sobre el bien tratado. Este asunto es trascendente tanto para la determinación del Dp como para la del Da.

La encuesta recopiló datos de orden demográfico, como para definir el perfil social del oferente, además de la respuesta en formato abierto sobre la disponibilidad a aceptar un monto por renunciar a un supuesto beneficio de mantener en su dominio las tierras con araucarias. El resultado de la caracterización entregó un perfil generalizado como el que sigue: “... un 96\% de los dueños de araucarias se dedica a la ganaderia, el resto se distribuye en actividades de turismo y artesania. No se dedican comercialmente a la agricultura debido a las características climáticas de la zona, recordar que es cordillerana, y un suelo de baja calidad de dificil acceso." Una pregunta podría ser ¿Por qué no se dedican a la cosecha y venta de los piñones (fruto de la araucaria)? La respuesta radica en un asunto de costo-beneficio. La cosecha de piñones en forma competitiva tiene costos por sobre los ingresos de su comercialización $\left(100 \$ \mathrm{CH}^{4}\right.$ por kilo, con una recolección per-cápita de 30-50 kilos), o sea, beneficio neto negativo.

Las encuestas revelaron que los dueños no consideran utilizar los suelos con araucarias para comercializar con ellos, sino que prefieren dejar sus terrenos sin cuidado y emigrar con objetivos laborales a urbes más desarrolladas.

\section{- Valorización Contingente: Disponibilidad a Pagar: Caracterización de la Demanda}

En general, el estudio de valoración de bienes y servicios ambientales derivados al uso y calidad del agua, aplicando el método de valoración contingente, requiere de la aplicación de una encuesta, en la cual se consulta a las personas por su disposición a pagar, disposición a aceptar frente a una mejora, deterioro en la cantidad o calidad del bien ambiental o calidad del

\footnotetext{
${ }^{3} \mathrm{El}$ investigador está consciente que el panel de expertos de la NOAA recomienda utilizar la DAP antes que la DAA, dado que esta última para el caso de la demanda, dado resultados estilizados, entrega una estimación sobrevalorada (o sobreestimada), siendo la DAP más moderada. En este caso, se utiliza la DAA dado que se necesita saber la medida de bienestar que muestre a qué precios estarían dispuestos (los dueños) por dejar de percibir ingresos en otra actividad, es decir, por el lado de la oferta.

${ }^{4}$ La nomenclatura \$CH, significa: pesos chilenos.
} 
agua y en este caso particular de estudio la belleza escénica.

Para el diseño de la encuesta, optimización de la muestra y determinación de la disposición a pagar se siguen los pasos reportados por Rubilar (2008). En este sentido se utilizan preguntas con la forma de pregunta tipo referéndum (Arrow et al., 1993). En cuanto al diseño la referencia es Horton (2002). Para la optimización de la muestra se sigue a Rubilar (2008) y Cooper (1993). Este último autor, desarrolla un método utilizando datos de un premuestreo que se basa en un procedimiento iterativo que selecciona el diseño de la encuesta que minimiza el error cuadrático medio de la medida de bienestar. Para luego diseñar una matriz de pagos que se les oferta en una encuesta definitiva a los encuestados beneficiarios del servicio. Luego, a partir de las respuestas de las personas encuestadas, puede obtenerse la medida de bienestar y con ello la autoridad podrá contar con una medida aproximada de lo que las personas están dispuestas a pagar. Esto podria dar una idea al responsable de tomar decisiones sobre las posibles contribuciones que podrian hacer los agentes privados para acceder o mejorar la calidad de un bien ambiental.

A través de un análisis porcentual, aplicado a las respuestas obtenidas de los encuestados, es posible diagnosticar el grado de aceptación de este proyecto dentro de la muestra elegida y si se tiene la suficiente representatividad, incluso será posible hacer algunas inferencias sobre la población de estudio.

Para la obtención de la disposición a pagar se utiliza la metodologia de la Valoración Contingente (VC), dentro de la cual se aplica una técnica ampliamente conocida en el ámbito dominada por su aplicación empirica y no de una significativa contribución teórica (Rubilar, 2008). En particular, la que enfrenta a los individuos a poder optar a una mejora en la calidad ambiental de $\mathrm{q}_{0}$ a $\mathrm{q}_{1}$, por la cual deben pagar una cierta cantidad A Sin embargo, se debe tener presente que A no es necesariamente la verdadera disposición a pagar del individuo (la cual será designada como C), por ello la probabilidad de obtener una respuesta afirmativa por parte del encuestado será la probabilidad de que su verdadera disposición a pagar sea mayor o igual a la cantidad que se le está ofreciendo, o sea:

$$
\operatorname{Pr} o b(S i)=\operatorname{Pr} o b(C \geq A)
$$

Eligiendo una distribución para los errores (Probit y Logit) y asignando una forma funcional lineal a la función de utilidad indirecta es posible obtener los parámetros de esta última con la información obtenida a partir de las respuestas binarias.

Hanemann (1984) propone dar una forma funcional y luego obtener la diferencia de utilidad indirecta a estimar. Luego la función indirecta de utilidad de tipo lineal será:

$$
v=\alpha+\beta y+\varepsilon
$$


La diferencia de utilidad indirecta estará dada por:

$$
\Delta v=\alpha-\beta A
$$

Asimismo las medidas de bienestar, media y mediana de la Disposición a Pagar (DaP) serán calculadas, a través de la verdadera DaP denotada por "C".

$$
C=\frac{\alpha+\eta}{\beta}
$$

Derivándose luego las medidas de bienestar, que para el caso lineal se calculan de forma similar se tiene:

$$
\operatorname{Media}[E(C)]=\text { Mediana }=\frac{\alpha}{\beta}
$$

Luego, una vez estimado el modelo, se debe analizar la significancia de las variables y posteriormente si son consistentes con la teoria económica. Finalmente se determina los intervalos de confianza para la disposición a pagar.

\section{RESULTADOS}

\section{Disponibilidad a Aceptar}

Anteriormente se mencionó que se considera que los dueños de araucarias consideran como capital muerto sus terrenos con bosques nativos. Este simple asunto lleva a ver esta situación desde otra perspectiva. Si la posesión de araucarias no genera beneficios, dado que no se puede cortar para usufructuar de su madera y la cosecha de su fruto no es económica ni contablemente rentable, entonces el costo de asignarle un nuevo uso, costo de oportunidad, debiese ser cero, i.e. cualquier actividad que genere beneficios positivos entregaria rentabilidad económica más alta que si se mantiene el bien en statu-quo. Además, el beneficio seria capitalizado en su totalidad como se observa en las Figuras $\mathrm{N}^{\circ} 1$ y 2 . Se puede suponer dos formas generales para el comportamiento de los beneficios. Por un lado, decir que el mecanismo que se implemente para generar el servicio ambiental entregará el máximo beneficio obtenible desde el primer período en funcionamiento, como en la (Figura $\mathrm{N}^{\circ} 1$ ), desde un periodo $t_{0}$ en adelante obtendrá un ingreso $\mathrm{Ing}_{0}>0$, en términos reales, hasta obtener un beneficio neto del tamaño del área bajo la curva de ingreso del periodo $t_{0}$ al infinito ${ }^{5}$.

\footnotetext{
${ }^{5}$ Hipotéticamente si el parque continua hasta el tiempo infinito.
} 
Por otro lado se puede pensar que como todo instrumento nuevo y por primera vez aplicado tendrá un periodo de adaptación, desde el punto de vista social y político, por lo que podría presentar beneficios marginales decrecientes desde su creación hasta un periodo $t_{1}>t_{0}$ alcanzando un ingreso $I n g_{1}>0$ ( $/ n g_{0}$ podria ser igual a $I n g$,). El área achurada en ambos gráficos determina el beneficio privado neto de participar como oferente en el mercado de servicios ambientales implementado.

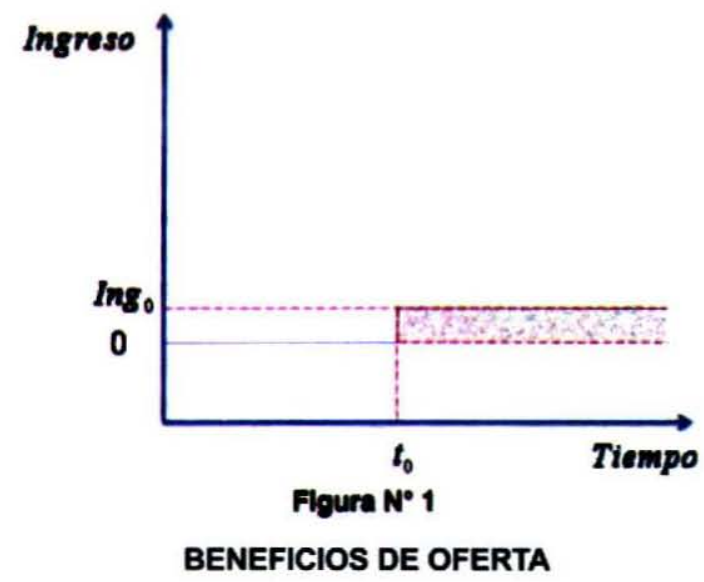

El pago mensual es el vehículo escogido para el instrumento y determinó una Disponibilidad a Aceptar de 200.000 \$CH por persona que pueda integrar la fuerza laboral, para ceder sus tierras a una administración comunitaria, para la implementación de un parque que proteja la salud de los bosques de araucarias.

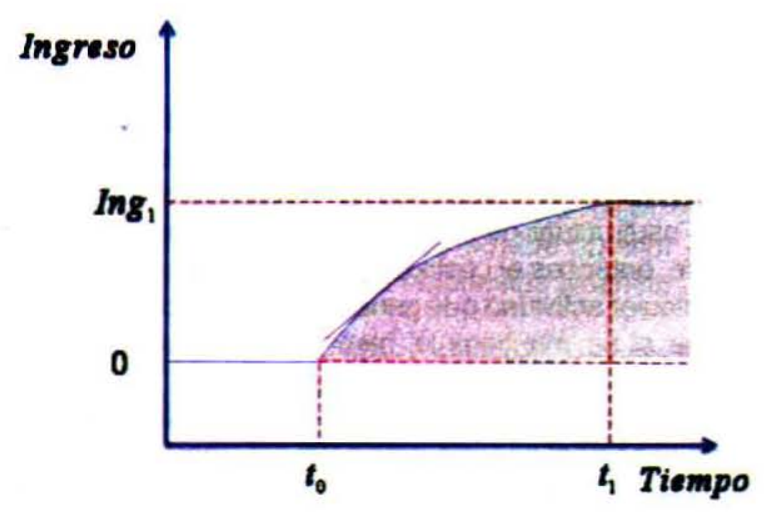

Figura $\mathrm{N}^{\circ} 2$

BENEFICIOS CAPITALIZADOS DE OFERTA 
La lógica de este resultado es sencilla. El efecto de no obtener rentas económicas de la posesión de suelos con bosques de araucarias genera migración laboral, i.e, traslado de fuerza laboral de una región a otra en forma permanente por razones de lucro. Esto debido a que las familias, al no percibir ingresos que permitan la sustentabilidad de la familia se van a vivir a ciudades con posibilidades de trabajo más variadas, pero recibiendo un ingreso promedio como el salario minimo legal de Chile.

La alternativa de emigrar ha sido cada vez más recurrente, llevando al despoblamiento de esta zona. En consecuencia, la implicancia relevante del Pago por Servicios Ambientales es la de generar un incentivo a estas familias para que permanezcan en sus dominios participando en actividades de conservación y mantención del bosque nativo centrado en la araucaria, dentro del marco de un parque forestal.

Finalmente, lo que queda es ofrecerle a estos potenciales oferentes rentabilidades mayores que las percibidas en otras ciudades, y estas rentabilidades no deben ser, necesariamente, considerablemente mayores que el sueldo mínimo real. En términos no específicos, se puede decir que el salario de indiferencia es el salario minimo real. Si el ingreso real es mayor que el minimo, la familia esta dispuesta a participar del PSA, hipotéticamente hablando. Mientras que si el ingreso real es menor que el mínimo, los individuos prefieren dejar sus tierras y emigrar a ciudades más grandes y atractivas.

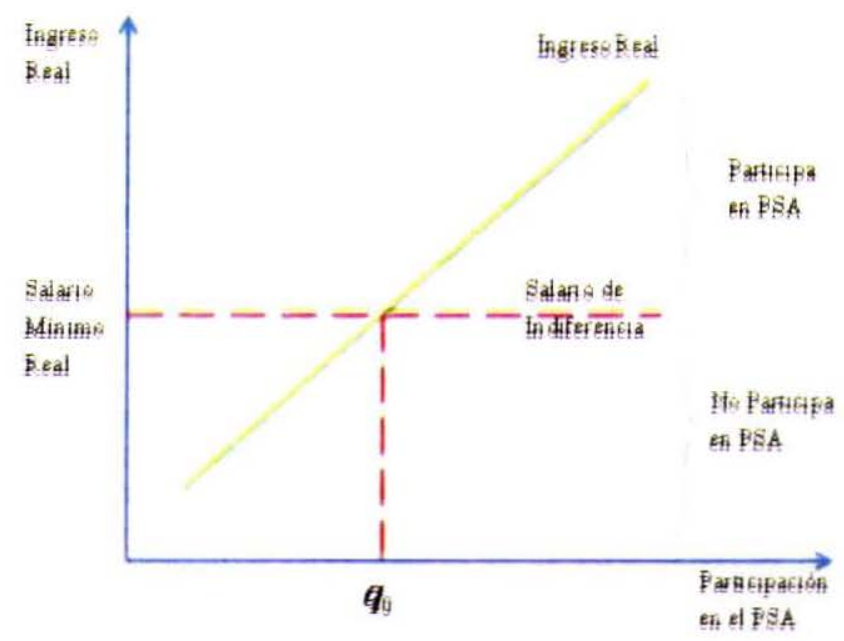


Se llama "Participación en el PSA" a la cantidad de esfuerzo aplicada en el trabajo del parque de conservación de araucarias y este esfuerzo puede estar determinado por la cantidad de miembros de la familia que participan en dicho trabajo, también puede ser la cantidad de horas-hombre dedicadas al parque, como también la calidad de este trabajo. Estas variables serian las mismas observadas en un trabajo en ciudades grandes. Finalmente genera la idea de que la curva de ingreso real, suponiendo linealidad y la misma pendiente para todas las familias por simplicidad, para algunas personas sería más baja y para otras más altas, relativamente. Entonces, como se grafica en la Figura $\mathrm{N}^{\circ} 4$, la decisión de participar en labores del parque depende del ingreso real ofrecido y de las variables agregadas en el esfuerzo. Donde el punto $\left(q_{0}=\right.$ Sal.Minimo $)$ es de indiferencia para los agentes oferentes.

Ahora, para caracterizar toda la oferta es necesario considerar sólo la suma horizontal de las ofertas individuales desde el punto de indiferencia antes mencionado.

Un aspecto importante a resaltar es que la oferta no está determinada por la cantidad de tierras que se posea ni por la cantidad de araucarias que se posea, ni menos por la densidad del bosque nativo, sino solamente por el ingreso real ofrecido, suponiendo que no existe ilusión monetaria. Es decir, a mayor cantidad de araucarias, los dueños no percibirán mayores beneficios que los dueños con menos forestación, sin embargo, por el esfuerzo incluido al ingreso ofrecido se podrán optar a mayores rentabilidades o a menores según las capacidades de cada agente.

Considerando sólo desde el salario de indiferencia hacia arriba individualmente, dado un precio, se genera la oferta agregada del mercado de servicios ambientales.

Este resultado no deja de ser bastante atractivo desde la perspectiva politica, dado que la cantidad de dueños a los que se les debe pagar por la entrega del cuidado de sus bosques es tan baja y además el precio que exigen es, de momento, cercano al sueldo mínimo real, concluyendo a priori con la idea de que la aplicación de esta metodologia es económicamente factible para una demanda determinada, recomendándose. Sin embargo, la ampliación y profundización de esta investigación, es necesaria.

\section{Disposición a Pagar}

Luego de aplicar la pre encuesta con formato abierto, a una muestra total de 60 personas, en la ciudad de Temuco, Chile, se utilizaron los tests N-Q Plot (visualización gráfica), asimetria y kurtosis para determinar la distribución de la $\mathrm{Dp}$ en el pre muestreo, y luego analizando los cuartiles en la muestras se ve que están distribuidos semejantes a la situación hipotética (distribución normal). Por tanto, a través del análisis de los tests se determinó que la Dp tiene una distribución simétrica, logit. Posteriormente, con el tamaño de la muestra "N" dado, igual a 380 (números de encuestas), se calculó el vector de cantidades de pago ofrecidas "m" y las submuestras " $n$ " para cada cantidad de pago ofrecida "Ai". Asi se determinó la matriz de pagos que se incorpora en la encuesta (Rubilar, 2008).

Para la estimación de las medidas de cambio en el bienestar media y mediana, se utilizó forma funcional lineal Hanemann (1984). En las estimaciones, la variable dependiente 
denominada $\mathrm{Dp}$, está representada por las respuestas si/no de disposición a pagar declaradas por los individuos. Por otro lado, las variables independiente, utilizada fue: pago ofertado (BID).

Las variables estadisticamente significativas al $10 \%$ y al $5 \%$, fueron pago ofrecido. Sin embargo, en este estudio se considera como única variable explicativa el vector de cantidades ofrecidas, esto con el fin de poder estimar la medida de bienestar con estimaciones paramétricas simples. Al analizar las variables significativas, la variable BID, presenta signo negativo, lo cual la hace consistente con la teoria económica, porque a mayor cantidad de pago ofrecido, existe menor Dp por parte de los encuestados.

En relación a la bondad de ajuste de los modelos, analizando los indicadores de selección del programa estadistico utilizado $R^{2}$ Mc Fadden, cuyos valores fueron bajos para la forma funcional lineal y Akaike, cuyos valores indican que el modelo con distribución logit es levemente mejor. Sin embargo, el porcentaje de predicciones correctas, es decir, la cantidad de aciertos al predecir el comportamiento de la variable dicotómica de respuesta (Dp) coincide en ambos modelos.

\section{Cuadro $\mathrm{N}^{\circ} 2$ \\ ESTIMACIÓN DE LOS COEFICIENTES DE LA DIFERENCIA DE LA FUNCIÓN INDIRECTA DE UTILIDAD}

SEGÚN SUS DOS FORMAS FUNCIONALES - CIUDAD DE TEMUCO

\begin{tabular}{|c|c|c|}
\hline & \multicolumn{2}{|c|}{$\begin{array}{l}\text { Forma Funcional Lineal Hanemann (1984) } \\
\qquad \Delta v=\alpha-\beta A_{i}\end{array}$} \\
\hline & \multicolumn{2}{|c|}{ Distribución } \\
\hline & Probit & Logit \\
\hline Constante & $\begin{array}{c}0,2554 \\
(1.920)^{\star}\end{array}$ & $\begin{array}{c}0,4258 \\
(1,974)^{\star}\end{array}$ \\
\hline $\operatorname{Bid}\left(A_{1}\right)$ & $\begin{array}{l}-0,00023 \\
(-4.370)^{\star \star *}\end{array}$ & $\begin{array}{l}-0,0004 \\
(-4,316)^{\star \star \star *}\end{array}$ \\
\hline$R^{2}$ McFadden & 0,0383 & 0,0388 \\
\hline Akaike (AIC) & 1,30504 & 1.30437 \\
\hline Chi Cuadrado & 19,57378 & 19,82815 \\
\hline$\%$ Pred. Corr. & 78,95 & 78,95 \\
\hline
\end{tabular}

(Fuente: Elaboración propia en base a los datos obtenidos de las regresiones corridas)

Valores " $t$ ", significancia estadistica ()$^{*}$ al $10 \%()^{\cdots *}$ al $1 \%$. 
Si se analiza la medida de cambio en el bienestar "mediana" obtenida, los valores de esta para la población de Temuco, bajo el enfoque de estimación paramétrica, recordando que para la forma funcional lineal la media y mediana coinciden, se obtuvo los valores de $991 \mathrm{CHS}$ y $1046 \mathrm{CH} \$$ por mes.

\section{Cuadro $\mathrm{N}^{\circ} 3$ \\ MEDIDAS DE CAMBIO EN EL BIENESTAR OBTENIDAS A TRAVÉS DE ESTIMACIONES PARAMÉTRICAS, \\ MODELO DE DIFERENCIA DE LA FUNCIÓN INDIRECTA DE UTILIDAD PARA LOS HABITANTES DE TEMUCO.}

\begin{tabular}{|c|c|c|}
\hline \multirow{2}{*}{ 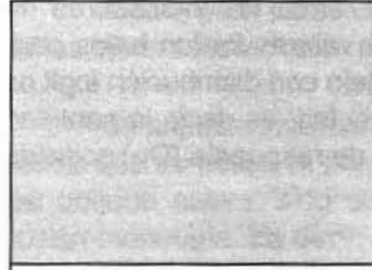 } & \multicolumn{2}{|c|}{$\begin{array}{l}\text { Forma Funcional Lineal Hanemann (1984) } \\
\qquad \Delta v=\alpha-\beta A_{i}\end{array}$} \\
\hline & $\begin{array}{l}\text { Probit } \\
\mathrm{CH} \$ / \text { mes }\end{array}$ & $\begin{array}{l}\text { Logit } \\
\mathrm{CH} \text { /mes }\end{array}$ \\
\hline Media* & $\begin{array}{c}991 \\
{[460-1522]^{* *}}\end{array}$ & $\begin{array}{c}1046 \\
{[633-1461]^{* *}}\end{array}$ \\
\hline Mediana* & $\begin{array}{c}991 \\
{[460-1522]^{* *}}\end{array}$ & $\begin{array}{c}1046 \\
{[633-1461]^{* *}}\end{array}$ \\
\hline
\end{tabular}

(Fuente: Elaboración propia. Valor de las medidas de cambio en el bienestar en CH\$/mes)

[1] Intervalos de confianza obtenidos a través del procedimiento estándar, () ${ }^{*}$ al $5 \%$

De los encuestados, el 53,2\% están dispuestos a pagar para asegurar que las Áreas con Bosques de Araucarias no protegidas se destinen a un parque de áreas de Belleza Escénica y Conservación. Aun cuando se le hacia presente que al pagar este monto de dinero le estará restando la posibilidad de adquirir otros bienes o servicios, además él (encuestado) y su familia tendria que pagar esta cantidad en cada periodo. Por otro lado, la razón principal del porcentaje de la población que no está dispuesta a pagar, esgrime que el gobierno debe pagar por esto con un $21 \%$ (del total de la muestra).

En particular, al medir el grado de importancia de las principales razones de disposición a pagar se destaca: Se debe proteger estas áreas para futuras generaciones (no uso, herencia); debe existir, porque además alli habita gran diversidad de vegetación y animales (existencia); y el bosque entrega aire limpio, abastecimiento de agua, recreación y turismo (uso indirecto). Estas alternativas en cada una de ellas representan un $40 \%$ de los encuestados.

\section{Precio de Equilibrio y Factibilidad de un PSA}

Al considerar en conjunto los dos apartados precedentes se puede analizar cómo se ha plasmado empíricamente este mercado hipotético de un parque de conservación de belleza escénica. Asi es posible desarrollar dos considerandos que conceptualizan un "seudo-precio 
de equilibrio" y factibilidad de conformar un proyecto de PSA.

Cuando se trabaja en la determinación de un precio se debe tener el cuidado de especificar claramente el tipo de bien que se transaria en este mercado hipotético (Rubilar, $2008)^{6}$. Las araucarias por un lado son un bien privado dado que pertenecen a propietarios particulares y al consumirlos comercialmente se agotarian, pero si se considera que por ley son un Monumento Natural pasa a tener igualmente otra clasificación dentro de los denominados bienes públicos. Asi entonces su consumo es simultáneo por varios consumidores y este "no se debiera agotar". Claramente se está en presencia de un conflicto de usos y/o de no-usos, cuyo análisis escapa de las pretensiones de este estudio, pero que sienta las bases para desarrollar en mayor profundidad a futuro la naturaleza de las mismas.

Al considerar la demanda de este tipo de bienes se puede esperar que los consumidores, ciudadanos de Temuco, estén dispuestos aceptar pagar por recibir este beneficio. Este pago si bien es cierto es mayor al que se deberia pagar a los propietarios, permite razonar la factibilidad de implementar este tipo de proyectos. Aún cuando, la literatura advierte que los encuestados tienden a sobre estimar su disposición a pagar, el margen obtenido lleva a concluir en la factibilidad de la misma. Pero se deja el alcance que la implementación de este tipo de proyecto se limita cuando se consideran otras superficies que tiene la especie en el pais y/o cuando se consideran otros tipos de consumidores, distinto a los ciudadanos de Temuco, como pueden ser turistas extranjeros o nacionales. Esto sin duda amerita incrementar el número de encuestas con modificaciones del tipo de muestreo, análisis de preferencias y resultados de modelaciones econométricas.

Por el lado de la oferta, los dueños presentan un comportamiento bastante estándar, es decir, sus medidas de bienestar no se alejan en general de lo que perseguiria cualquier agente económico racional, siguiendo una conducta atractivamente apegada a la teoría microeconómica. En otras palabras, si se logra cubrir el costo de oportunidad de los dueños de quedarse en sus propias tierras para administrarlas como un parque forestal, estos tendrian suficientes (en términos no estrictos) incentivos económicos. Dado que, como se definió anteriormente, el umbral de indiferencia está determinado por el salario real mínimo, esto por la baja calificación laboral de los dueños, y además, la cantidad de dueños es tan pequeña que el costo que se debiera cubrir para mantener a los dueños satisfechos seria cercano a $\mathrm{CH} \$ 2.000 .000$. $^{7}$ Esto dado un diseño contractual similar al que recibirian en una ciudad receptora. Con este monto mensual para la mantención de un bosque de tamaño considerable, se puede conjeturar que la implementación de un programa de PSA es factible dados los resultados de la Dp y la cantidad de familias que participarian del pago.

De los reportes de este estudio se puede clarificar que la factibilidad de implementar un proyecto de PSA y en el caso particular de la belleza escénica puede ser posible si se consideran los siguientes aspectos.

La disposición a pagar de la población es bastante realista dada el porcentaje de

\footnotetext{
- Para una definición tentativa de bienes véase Rubilar (2008).

'Bajo el supuesto de 2 miembros que pertenecen a la fuerza laboral por familia-dueño, a $\mathrm{CH} \$ 200.000$ de salario mínimo nominal (o real si el indice inflacionario es 1), todo esto por 5 dueños o familias.
} 
respuestas afirmativas y del nivel de significancia del parámetro pago ofertado (BID).

Las estimaciones de $\mathrm{CH} \$$ en el análisis de la medida de cambio en el bienestar permite inferir que el proyecto es económicamente factible al existir un trade-off entre los bosques protegidos de araucaria y la demanda por beneficios ambientales.

El éxito de este proyecto pasa por considerar algunas restricciones legales en cuanto a la tenencia de la tierra (títulos de dominio), ley indigena (algunos propietarios son de la etnia pehuenche) y de la existencia de posibles sitios arqueológicos y/o cementerios indigenas y otras leyes relacionadas (código de aguas, ley de bosque, entre otras).

Otro factor básico para su implementación debe considerar una institucionalidad nueva, creible y sustentable en el tiempo como por ejemplo una Fundación.

\section{CONCLUSIONES}

La principal conclusión es que se observa perfectamente factible implementar el Pago por Servicios Ambientales a bosques de Araucaria araucana.

Los dueños de los predios con araucarias, tienen un comportamiento restringido por las oportunidades de mercado, son de baja calificación y tienen restricciones sobre sus tierras. Ello favorece o motiva fuertemente desde el punto de vista económico la aceptación de la presente propuesta. Pero más aún, dada su cultura y su arraigo sugiere considerar un agente cautivo, por el lado de la oferta, hacia ese tipo de proyecto de PSA.

Los ciudadanos de Temuco presentan un nivel de aceptación bastante conservador, lo que significaria que le asigna mucha importancia al tema de la conservación y atractivo de la especie araucaria. Pero con una clara limitante que su aceptación esta condicionada al nivel o monto de pago que deben efectuar para beneficiarse en contraposición de la adquisición de otros bienes por un lado y por otro en que seria el gobierno el responsable de la conservación de la especie araucaria.

Finalmente, la implementación de un proyecto de PSA orientado hacia la belleza escénica estará restringida a los siguientes aspectos:

-Una institucionalidad fuerte, creíble y sustentable en el tiempo.

-Aspectos legales, económicos, sociales y culturales asociado al pueblo Pehuenche.

-Estudios bioeconómicos y de distribución geográfica de la especie en Chile con objetivos de un proyecto de PSA.

Como último comentario y sugerencia al referirse a este tipo de estudios, este primer esfuerzo en Chile sugiere la necesidad de desarrollar una mayor cantidad de trabajos e implementación de procesos formadores de PSA, lo que se justifica por la gran diversidad del ambiente natural y la distribución geográfica de las especies nativas, tanto de flora como de fauna. 


\section{REFERENCIAS}

Antle, John M.; Valdivia, Roberto O., 2006. "Modelling the supply of ecosystem services from agriculture: a minimum-data approach". The Australian Journal of Agricultural and Resource Economics, 50: 1-15.

Arrow, K., R. Solow, P. Portney, E. Leamer, R. Radner y H. Schuman, 1993. "Report of the NOAA panel on contingent valuation". Federal Register 58. 4601-14. Washington D.C. U.S.A. 1993.

Bayon, R., 2004. "Making environmental marketswork: lessons from early experience with sulfur, carbon and wetlands." Forest Trends, Washington, D.C.

Claassen, Roger; Cattaneo, Andrea; Johansson, Robert, 2005. "Cost-Effective Design of Agri-Environmental Payment Programs: U.S. Experience in Theory and Practice" ZEF-CIFOR workshop on payments for environmental services in developed and developing countries. Titisee, Germany, June 16-18, 2005.

Cooper, Joseph C., 1993. "Optimal Bid Selection for Dihotomous Choice Contingent Valuation Surveys", JEEM, 24, 25-40. 1993.

De Hek, Simone; Kiersch, Benjamin; Mañon, Alejandro, 2004. "Aplicación de Pagos por Servicios Ambientales en manejo de Cuencas Hidrográficas: lecciones de experiencias recientes en América Latina". Organización de las Naciones Unidas para la Agricultura y la Alimentación (FAO), Oficina Regional de la FAO para América Latina y el Caribe.

Engel, Stefanie; Palmer, Charles, 2008. "Payments for environmental services as an alternative to logging under weak property rights: The case of Indonesia". Ecological Economics 65. 799-809.

Ferraro, Paul J., 2005. "Asymmetric Information and Contract Design for Payments for Environmental Services". Preliminary Draft, Department of Economics, Andrew Young School of Policy Studies, Georgia State University, Atlanta.

Hanemann, Michael W., 1984. "Welfare Evaluations in Contingent Valuation Experiments with Discrete Responses". American Journal of Agricultural Economics 66: 322-241.

Horton, B., Colarullo, G., Bateman, I.J \& Peres, C., 2002. "Evaluating non - User Willingness to pay for a large - scale conservation program in Amazonia: a UK/Italian contingent valuation study". CSERGE Working Paper ECM 02-01, Centre for social and Economic Research on the Global Environment, University of East Anglia and University College London, UK.

Karsenty, A., 2004. "Des rentes contre le d'eveloppement? Les nouveaux instruments d'acquisition mondiale de la biodiversit'e et l'utilisation des terres dans les pays tropicaux." Mondes en D'eveloppement 127:1-9.

Landell-Mills, Natasha; Porras, Ina T., 2002. "Silver bullet or fools' gold? A global review of 
markets for forest environmental services and their impact on the poor". International Institute for Environment and Development (IIED), London.

Myers, Norman, 1996. "Environmental Services of Biodiversity". Proceedings of the National Academy of Sciences of the United States of America, Vol. 93, No. 7, pp. 2764-2769.

Romero, C.; G. I. Andrade, 2004. "International conservation organizations and the fate of local tropical forest conservation initiatives." Conservation Biology 18:578-580.

Rubilar, Marco Ant., 2008. "Breve revisión del procedimiento para estimación de la disposición de pago para la valoración económica." Documento de trabajo. INFOR- Universidad de Concepción. 1-20.

Sierra, Rodrigo; Russman, Eric. "On the efficiency of environmental service payments: A forest conservation assessment in the Osa Peninsula, Costa Rica". Ecological Economics 59 131-141. 2006.

Siikamäki, J.; Layton, D.F., 2006. "Potential cost-effectiveness of incentive payment programs for biological conservation." Discussion Paper 06-27, Resources For the Future, Washington DC.

Sokolow, A. D.; Zurbrugg, A., 2003. "A national view of agricultural easement programs." Center for Agriculture in the Environment, American Farmland Trust, Davis, California.

Swallow, Brent; Meinzen-Dick, Ruth; van Noordwijk, Meine, 2005. "Localizing demand and supply of environmental Services: interactions with property rights, Collective action and the welfare of the poor". CAPRi Working Paper \# 42.

Vogel, J., 2002. "Markets or metaphors? A sustainable livelihoods approach to the management of environmental services: two cases from Ecuador." International Institute for Environment and Development, London.

Wunder, Sven, 2005. "Payments for environmental services: Some nuts and volts". CIFOR, Occasional Paper $\mathrm{N}^{\circ} 42$.

Wunder, Sven; Engel, Stefanie; Pagiola, Stefano, 2008. "Taking stock: A comparative analysis of payments for environmental services programs in developed and developing countries". Ecological Economics 65, 834-852.

Wünscher, Tobias; Engel, Stefanie; Wunder, Sven, 2007. "Spatial targeting of payments for environmental services: A tool for boosting conservation benefits". Ecological Economics $65822-833$.

Wünscher, T.; Engel, S.; Wunder, S., 2006. "Payments for environmental services in Costa Rica: increasing efficiency through spatial differentiation." Quarterly Journal of International Agriculture 45 (4), 319-337. 